Pacific Journal of Mathematics

DECOMPOSITION THEOREMS FOR 3-CONVEX SUBSETS OF 


\title{
DECOMPOSITION THEOREMS FOR 3-CONVEX SUBSETS OF THE PLANE
}

\author{
MARILYN BREEN
}

Let $S$ be a 3-convex subset of the plane. If $(\operatorname{cl} S \sim S) \subseteq$ int $(\operatorname{cl} S)$ or if $(\operatorname{cl} S \sim S) \subseteq$ bdry $(\operatorname{cl} S)$, then $S$ is expressible as a union of four or fewer convex sets. Otherwise, $S$ is a union of six or fewer convex sets. In each case, the bound is best possible.

1. Introduction. Let $S$ be a subset of $R^{d}$. Then $S$ is said to be 3-convex iff for every three distinct points in $S$, at least one of the segments determined by these points lies in $S$. Valentine [2] has proved that for $S$ a closed, 3-convex subset of the plane, $S$ is expressible as a union of three or fewer closed convex sets. We are interested in obtaining a similar decomposition without requiring the set $S$ to be closed. The following definitions and results obtained by Valentine will be useful.

For $S \subseteq R^{d}$, a point $x$ in $S$ is a point of local convexity of $S$ iff there is some neighborhood $U$ of $x$ such that, if $y, z \in S \cap U$, then $[y, z] \subseteq S$. If $S$ fails to be locally convex at some point $q$ in $S$, then $q$ is called a point of local nonconvexity (lnc point) of $S$.

Let $S$ be a closed, connected, 3-convex subset of the plane, and let $Q$ denote the closure of the set of isolated lnc points of $S$. Valentine has proved that for $S$ not convex, then card $Q \geqq 1, Q$ lies in the convex kernel of $S$, and $Q \subseteq$ bdry $(\operatorname{conv} Q)$. An edge of bdry $(\operatorname{conv} Q)$ is a closed segment (or ray) in bdry (conv $Q$ ) whose endpoints are in $Q$. We define a leaf of $S$ in the following manner: In case card $Q \geqq 3$, let $L$ be the line determined by an edge of bdry (conv $Q), L_{1}, L_{2}$ the corresponding open halfspaces. Then $L$ supports conv $Q$, and we may assume conv $Q \subseteq \operatorname{cl}\left(L_{1}\right)$. We define $W=\operatorname{cl}\left(L_{2} \cap S\right)$ to be a leaf of $S$. For $2 \geqq$ card $Q \geqq 1$, constructions used by Valentine may be employed to decompose $S$ into two closed convex sets, and we define each of these convex sets to be a leaf of $S$.

By Valentine's results, every point of $S$ is either in conv $Q$ or in some leaf $W$ of $S$ (or both), and every leaf $W$ is convex. Moreover, Valentine obtains his decomposition of $S$ by showing that for any collection $\left\{s_{i}\right\}$ of disjoint edges of bdry $(\operatorname{conv} Q)$, with $\left\{W_{i}\right\}$ the corresponding collection of leaves, conv $Q \cup\left(\cup W_{i}\right)$ is closed and convex.

Finally, we will use the following familiar definitions: For $x, y$ in $S$, we say $x$ see $y$ via $S$ iff the corresponding segment $[x, y]$ lies in $S$. A subset $T$ of $S$ is visually independent via $S$ iff for every 
$x, y$ in $T, x$ does not see $y$ via $S$.

Throughout the paper, $S$ will denote a 3-convex subset of the plane, $Q$ the closure of the set of isolated lnc points of $\operatorname{cl} S$.

2. Preliminary lemmas. The following sequence of lemmas will be useful in obtaining the desired representation theorems. We begin with an easy result.

Lemma $1 . \mathrm{Cl} S$ is 3-convex.

Proof. Let $x, y, z$ be distinct points in $\operatorname{cl} S$ and select disjoint sequences $\left(x_{i}\right),\left(y_{i}\right),\left(z_{i}\right)$ in $S$ converging to $x, y, z$ respectively. For each $i$, one of the corresponding segments is in $S$, and for one pair, say $x$ and $y$, infinitely many of the segments $\left[x_{\imath}, y_{\imath}\right]$ lie in $S$. Since these segments converge to $[x, y],[x, y]$ lies in $\mathrm{cl} S$.

The remaining lemmas are technical in nature. Lemmas 2, 3, and 4 reveal various pleasant features of int $(\mathrm{cl} S) \sim S$, while 5 and 6 are concerned with lnc points of $\mathrm{cl} S$.

Lemma 2. If $p \in \operatorname{int}(\operatorname{cl} S) \sim \operatorname{ker}(\operatorname{cl} S) \neq \varnothing$, then $p \in S$.

Proof. Since $p \notin \operatorname{ker}(\operatorname{cl} S)$, there is some point $x$ in $\mathrm{cl} S$ for which $[x, p] \nsubseteq \operatorname{cl} S$. Moreover, $x$ may be chosen in $S$ (for if $p$ saw every member of $S$ via cl $S$, then $p$ would see every member of cl $S$ via $\operatorname{cl} S$ and $p$ would lie in $\operatorname{ker}(\mathrm{cl} S)$ ).

There is a convex neighborhood $N$ of $p$, no point of which sees $x$ via cl $S$, with $N \subseteq \operatorname{int}(\mathrm{cl} S)$. For any $s, t$ distinct points in $N \cap S$, necessarily $[s, t] \subseteq S$ by the 3-convexity of $S$, so $N \cap S$ is convex. Since $N \subseteq \operatorname{int}(\operatorname{cl} S), \quad p$ is interior to some triangle conv $\{w, y, z\}$ with vertices belonging to $N \cap S$. Then since $N \cap S$ is convex, conv $\{w, y, z\} \subseteq S$, and $p \in S$. In fact, $p \in \operatorname{int} S$.

Corollary. If $p \in \mathrm{cl} S \sim S$, then either $p \in$ bdry $(\mathrm{cl} S)$ or $p \in$ $\operatorname{ker}(\mathrm{cl} S)$ (or both).

Lemma 3. Let $T \neq \varnothing$ be the set of points $p$ of $\mathrm{cl} S \sim S$ for which $p \notin$ bdry (cl $S)$. Then every connected component of $T$ is either an isolated point of $\mathrm{cl} S \sim S$ or an interval. Moreover, there can be at most one isolated point, and all components of $T$ lie on a common line.

Proof. If $T$ is a singleton point, the result is immediate, so assume that $T$ contains at least two distinct points $x, y$. Let $L(x, y)$ denote 
the line determined by these points. It is clear that not both $x$ and $y$ can be isolated in cl $S \sim S$, for otherwise, since $x, y \in \operatorname{int}(\operatorname{cl} S)$, it would be easy to select three points of $S$ on $L(x, y)$ visually independent via $S$.

Again using the 3-convexity of $S, L(x, y) \cap S$ has at most two components, and $L(x, y) \cap T \leqq$ ker (cl $S$ ) has at most three components. By an earlier argument, at most one component of $L(x, y) \cap T$ is an isolated point, and clearly each component is either an isolated point or an interval.

To complete the proof, it suffices to show that $T \subseteq L(x, y)$. Let $t \in \operatorname{int}(\mathrm{cl} S) \sim L(x, y)$ to show $t \notin T$. Since $L(x, y) \cap T$ contains at most one isolated point, $L(x, y) \cap T$ contains at least one interval $(r, s) \subseteq \operatorname{int}(\mathrm{cl} S)$, and we may choose some point $u$ in $S$ for which $(u, t)$ cuts $(r, s)$. Then select a convex neighborhood $N$ of $t, N \subseteq$ int (cl $S)$, so that for every $q$ in $N,(u, q)$ cuts $(r, s)$. By techniques similar to those used in the proof of Lemma $2, N \cap S$ is convex and $t \in S$. Hence $t \notin T$ and $T \subseteq L(x, y)$.

LEMma 4. If $\mathrm{cl} S \sim S$ contains an interval $(r, s)$ disjoint from bdry (cl $S)$, then every lne point of $\mathrm{cl} S$ lies on $L(r, s)$.

Proof. Assume that for some lnc point $t$ of $\mathrm{cl} S, t \notin L(r, s)$. As in the proof of Lemma 3 , choose a point $u$ and a neighborhood $N$ of $t$ so that $u$ sees no point of $N \cap S$ via $S$. Since $t$ is an lnc point of cl $S, N$ contains points $v, w$ in $S$ which are visually independent via $S$. Hence $u, v, w$ are visually independent via $S$, a contradiction, and $t$ must lie on $L(r, s)$.

Lemma 5. If $p$ is in $\operatorname{ker}(\mathrm{cl} S)$ and $q, r$ are in $Q$, then $q \notin(p, r)$ (where $p, q, r$ are distinct points).

Proof. Assume, on the contrary, that the points are collinear, with $p<q<r$. Let $L$ denote the line containing $p, q, r, L_{1}, L_{2}$ the corresponding open halfspaces. Since $p \in \operatorname{ker}(\operatorname{cl} S)$ and $\operatorname{cl} S$ is not convex, there must be some point $x$ of cl $S$ not on $L$, say in $L_{1}$. Our hypothesis implies that cl $S$ is connected, so by [2], Corollary 1, $r \in \operatorname{ker}(\mathrm{cl} S)$, and the triangle conv $\{p, x, r\}$ has its boundary in cl $S$. It is easy to see that the closed, 3-convex set cl $S$ is simply connected, so conv $\{p, x, r\} \subseteq \operatorname{cl} S$. Thus since $q$ is an lnc point for cl $S$, there must be some point $y$ of $\operatorname{cl} S$ in $L_{2}$, conv $\{p, y, r\} \subseteq \operatorname{cl} S$, and $q$ cannot be an lnc point for cl $S$, clearly impossible. Our assumption is false, and $q \notin(p, r)$.

CoRollary. No three members of $Q$ are collinear. 
Lemma 6. If $p \in \operatorname{conv} Q, q \in Q, q \neq p$, and $W_{1}, W_{2}$ are leaves of cl $S$ containing $q$, then $W_{1}, W_{2}$ are in opposite closed halfspaces determined by $L(p, q)$.

Proof. Clearly the hypothesis implies that $\operatorname{cl} S$ is connected and that card $Q \geqq 2$. If card $Q=2$, the result is an immediate consequence of an argument used by Valentine (Case 2, Theorem 3 of [2]), so we may assume that card $Q \geqq 3$. Let $r$ lie on the edge of bdry (conv $Q)$ which defines $W_{1}, r \neq q$. If $r \in L(p, q) \equiv L$, then by the definition of $W_{1}$, it is obvious that $W_{1}$ is in one of the closed halfspaces determined by $L$, say cl $L_{1}$. Otherwise, without loss of generality, assume that $r$ is in the open halfspace $L_{1}$. Clearly $p$ and $W_{1}$ are separated by $L(r, q)$. Now if any point $x$ of $W_{1}$ lay in $L_{2}$, then $q$ would lie interior to the triangle conv $\{p, x, r\} \subseteq \mathrm{cl} S$, and $q$ could not be an lnc point for cl $S$, a contradiction. Hence $W_{1}$ lies in $\mathrm{cl} L_{1}$ in either case.

Since $W_{1} \cup \operatorname{conv} Q$ is convex (by Valentine's results) and $q$ is an lnc point for cl $S, W_{2}$ necessarily contains points in $L_{2}$, and $W_{2} \subseteq$ cl $L_{2}$, finishing the proof.

3. Decomposition theorems. With the preliminary lemmas behind us, we begin to investigate conditions under which $S$ may be represented as a union of four or fewer convex sets, dealing primarily with the case for $(\operatorname{cl} S \sim S) \subseteq \operatorname{int}(\operatorname{cl} S)$.

The first theorem, allowing us to restrict attention to the case for $\operatorname{cl} S=\operatorname{cl}($ int $S)$, will be helpful later.

THEOREM 1. If $\mathrm{cl} S \neq \mathrm{cl}$ (int $S$ ), then $S$ is a union of two or fewer convex sets.

Proof. Without loss of generality, assume $S$ is connected, for otherwise the result is trivial. Let $x \in S \sim \operatorname{cl}$ (int $S$ ) $\neq \varnothing$, and let $N$ be a convex neighborhood of $x$ disjoint from int $S$. Since $S$ is connected, $x$ is not an isolated point of $S$, and it is clear that $N \cap S$ contains at least one segment.

We examine the maximal segments of $N \cap S$ (i.e., the segments which are not proper subsets of segments in $N \cap S)$. It is easy to show that $N \cap S$ has at most two maximal segments, for otherwise, the 3-convexity of $S$ together with the simple connectedness of cl $S$ would yield an open region in $\operatorname{cl} S \cap N$. Since by Lemma 3 the points of int (cl $S) \sim S$ are collinear, this would imply that $N \cap S$ has interior points, clearly impossible by our choice of $N$.

In case $N \cap S$ has exactly two maximal segments, an argument similar to the one above may be used to show that any point of $S$ 
lies on one of the corresponding lines, and $S$ is a union of two segments (possibly infinite). If $N \cap S$ has just one segment, let $K_{1}$ denote a maximal convex subset of $S$ containing it, and let $K_{2} \equiv \operatorname{conv}\left(S \sim K_{1}\right)$. Again using the facts that $N$ contains no interior points of $\mathrm{cl} S$ and cl $S$ is simply connected, it is not hard to show that $K_{2} \subseteq S$, and $S=K_{1} \cup K_{2}$, completing the proof.

Theorems 2 and 3 show that a decomposition is possible when (cl $S \sim S) \subseteq \operatorname{int~}(\mathrm{cl} S)$. There are two cases to consider, depending on the cardinality of $Q$.

Theorem 2. If $(\operatorname{cl} S \sim S) \cap$ bdry $(\operatorname{cl} S)=\varnothing$, and $\operatorname{card} Q=n$ for $n$ an odd integer, $n>1$, then $S$ is expressible as a union of four or fewer convex sets.

Proof. Clearly the hypothesis implies that $\mathrm{cl} S=\operatorname{cl}($ int $S$ ). By the Corollary to Lemma 2, cl $S \sim S \subseteq \operatorname{ker}(\mathrm{cl} S$ ), and by Lemma 3, every component of $\operatorname{cl} S \sim S$ is either an isolated point or an interval. Since card $Q \geqq 3$ and (by the corollary to Lemma 5) no three members of $Q$ can be collinear, Lemma 4 implies that cl $S \sim S$ cannot contain an interval. Hence $\mathrm{cl} S \sim S$ consists of exactly one isolated point $p$ in $\operatorname{ker}(\mathrm{cl} S)$.

Select $q \in Q$ in the following manner: If $p \in \operatorname{conv} Q$, choose $q \in Q$ so that the line $L(p, q)$ contains no other member of $Q$. (Clearly this is possible since card $Q$ is odd and no three members of $Q$ are collinear.) If $p \notin \operatorname{conv} Q$, let $\left\{e_{i}: 1 \leqq i \leqq n\right\}$ denote the edges of conv $Q,\left\{E_{i}: 1 \leqq\right.$ $i \leqq n\}$ the corresponding lines, with $\operatorname{conv} Q$ in the closed halfspace $\operatorname{cl}\left(E_{i 1}\right)$ for each $i$. Then $p \in E_{i 2}$ for exactly one $i$, for otherwise, if $p \in E_{12} \cap E_{22}$, then int $\operatorname{conv}\left(\{p\} \cup e_{1} \cup e_{2}\right)$ would contain an lnc point of cl $S$, clearly impossible since $\{p\} \cup e_{1} \cup e_{2} \subseteq \operatorname{ker}(\operatorname{cl} S)$ and conv $\left(\{p\} \cup e_{1} \cup e_{2}\right) \subseteq \mathrm{cl} S$. Thus we may choose some $q \in Q$ so that $p \in \operatorname{cl} E_{i 1}$ for each edge $e_{i}$ containing $q$. Then $(p, q)$ contains points of $\operatorname{conv} Q$. Since all points of $L(p, q) \cap \operatorname{conv} Q$ are on the open ray at $p$ emanating through $q$, Lemma 5 implies that $L(p, q)$ contains no other members of $Q$ (and in fact $p$ cannot lie on any line $E_{i}$ ).

To review, in either case we have chosen $q$ in $Q$ so that $L(p, q)$ contains no other member of $Q$ and $(p, q)$ contains points of conv $Q$. Letting $L_{1}, L_{2}$ denote distinct open halfspaces determined by $L=$ $L(p, q)$, define $A \equiv \operatorname{cl}\left(S \cap L_{1}\right), B \equiv \operatorname{cl}\left(S \cap L_{2}\right)$. If $W_{1}, W_{2}$ are leaves of cl $S$ containing $q$, then by Lemma $6, W_{1}$ and $W_{2}$ are in opposite closed halfspaces determined by $L$, say $W_{1} \subseteq \operatorname{cl~} L_{1}, W_{2} \subseteq \operatorname{cl} L_{2}$.

Let $R_{1}, R_{2}$ denote opposite closed rays at $p, R_{1} \cup R_{2}=L$, labeled so that $q \in R_{2}$. Each of $R_{1} \cap S, R_{2} \cap S$ is an interval by the 3-convexity of $S$. Points of $R_{1} \cap S$ necessarily lie in $A \cap B$, for otherwise 
$R_{1}$ would contain an lnc point of cl $S$, clearly impossible. If there are any points of $R_{2} \cap S$ not in $A \cap B$, without loss of generality we may assume such points lie in $W_{1}$ and hence in $A \sim B$. Then $R_{2} \cap S \subseteq A$.

By Case 4 in Theorems 2 and 3 of [2], $\operatorname{cl}\left(S \sim W_{2}\right)$ is a union of two closed convex sets $C_{1}, C_{2}$, selected as in Valentine's proof. Since $A=$ $\operatorname{cl}\left[\mathrm{cl}\left(S \sim W_{2}\right) \cap L_{1}\right], A$ is the union of the two closed convex sets $A_{1}, A_{2}$, where $A_{i}=\operatorname{cl}\left(C_{i} \cap L_{1}\right), i=1,2$. Moreover, $\left(R_{1} \cap S\right) \cup(p, q]$ lies in one of these sets, say $A_{1}$, and $R_{2} \sim(p, q]$ is either in $A_{1}$ or in $A_{2}$.

Using an identical argument for $B$ and $\operatorname{cl}\left(S \sim W_{1}\right)$, we may write $B$ as a union of two closed convex sets $B_{1}, B_{2}$ with $\left(R_{1} \cap S\right) \cup(p, q]$ in $B_{1}$, and $R_{2} \sim(p, q]$ disjoint from $B$.

At last, define sets $A_{1}^{\prime}, A_{2}^{\prime}, B_{1}^{\prime}, B_{2}^{\prime}$ in the following manner: If $\left(R_{2} \cap S\right) \sim(p, q] \subseteq A_{2}$, let

$$
\begin{array}{ll}
A_{1}^{\prime} \equiv A_{1} \sim R_{2}, & A_{2}^{\prime} \equiv A_{2} \sim R_{1}, \\
B_{1}^{\prime} \equiv B_{1} \sim R_{1}, & B_{2}^{\prime} \equiv B_{2} \sim R_{2} .
\end{array}
$$

And if $\left(R_{2} \cap S\right) \sim(p, q] \subseteq A_{1}$, let

$$
\begin{array}{ll}
A_{1}^{\prime} \equiv A_{1} \sim R_{1}, & A_{2}^{\prime} \equiv A_{2} \sim R_{2}, \\
B_{1}^{\prime} \equiv B_{1} \sim R_{2}, & B_{2}^{\prime} \equiv B_{2} \sim R_{1} .
\end{array}
$$

We assert that these are convex subsets of $S$ whose union is $S$ : Clearly each is a convex subset of $S$, and $S \sim L$ is contained in their union. For $\left(R_{2} \cap S\right) \sim(p, q] \subseteq A_{2}, R_{2} \cap S \subseteq A_{2}^{\prime} \cup B_{1}^{\prime}, R_{1} \cap S \subseteq A_{1}^{\prime}$. For $\left(R_{2} \cap S\right) \sim(p, q] \subseteq A_{1}, R_{2} \cap S \subseteq A_{1}^{\prime}, R_{1} \cap S \subseteq B_{1}^{\prime}$. Hence in either case $S \cap L$ is contained in the union of these sets, and $S=A_{1}^{\prime} \cup A_{2}^{\prime} \cup B_{1}^{\prime} \cup B_{2}^{\prime}$, completing the proof of the theorem.

Theorem 3. If $(\operatorname{cl} S \sim S) \cap$ bdry $(\operatorname{cl} S)=\varnothing$ and $\operatorname{card} Q=n \geqq 0$, where $n$ (possibly infinite) is not an odd integer greater than one, then $S$ is expressible as a union of four or fewer convex sets.

Proof. If $S$ is not connected, the result is trivial. Otherwise, by Theorem 3 of Valentine [2], cl $S$ may be expressed as a union of two or fewer closed convex sets $A, B$. Using Lemma 3 , let $L$ be a line containing cl $S \sim S, L_{1}, L_{2}$ the corresponding open halfspaces. Since $S$ is 3-convex and $A$ is convex, $S \cap A$ is 3-convex, and hence $(S \cap A) \cap L$ has at most two components, say $C_{1}, C_{2}$. Let $R_{1}, R_{2}$ denote opposite rays on $L$ with $C_{1} \subseteq R_{1}, C_{2} \subseteq R_{2}$.

Define

$$
\begin{aligned}
& A_{1} \equiv\left(A \cap S \cap \operatorname{cl} L_{1}\right) \sim R_{1}, \\
& A_{2} \equiv\left(A \cap S \cap \operatorname{cl} L_{2}\right) \sim R_{2} .
\end{aligned}
$$


Then $A_{1}, A_{2}$ are convex subsets of $S$ whose union is $A \cap S$.

Similarly define convex sets $B_{1}, B_{2}$ whose union is $B \cap S$. Clearly $S=A_{1} \cup A_{2} \cup B_{1} \cup B_{2}$, the desired result.

Corollary. If $(\mathrm{cl} S \sim S) \cap$ bdry $(\mathrm{cl} S)=\varnothing$, then $S$ is expressible as a union of four or fewer convex sets. The number four is best possible.

That the number four in the corollary is best possible is evident from Example 1.

ExAMPle 1. Let $S$ be the set in Figure 1, with $p \in S$. Then $S$ is not expressible as a union of fewer than four convex sets.

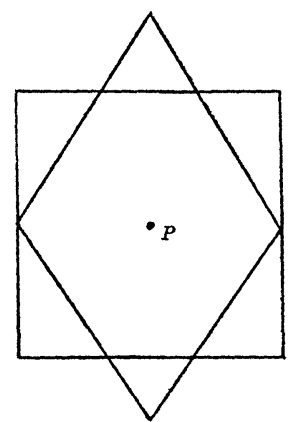

FigURE 1

The preceding theorems allow us to obtain the following decomposition for open sets.

THEOREM 4. If $S$ is open, then $S$ is expressible as a union of four or fewer convex sets. The result is best possible.

Proof. Let $T \equiv S \cup$ bdry (cl $S$ ). Applying arguments identical to those used in the proofs of Theorems 2 and $3, T$ is expressible as a union of four or fewer convex sets $A_{i}, 1 \leqq i \leqq 4$. Define $B_{i} \equiv$ $A_{i} \cap S, 1 \leqq i \leqq 4$. We assert that each $B_{i}$ is convex. The proof follows:

By Valentine's results, cl $S$ is expressible as a union of three or fewer closed convex sets $C_{j}, 1 \leqq j \leqq 3$, each consisting of an appropriate selection of leaves of $\mathrm{cl} S$, together with conv $Q$. Examining the proofs of Theorems 2 and 3 , it is clear that each $A_{i}$ may be considered as a subset of some $C_{j}$ set. Thus we may assume $B_{1} \subseteq$ $A_{1} \subseteq C_{1}$ for an appropriate $C_{1}$.

Let $x, y \in B_{1}$, and let $p \in(x, y)$ to show $p \in B_{1}$. If $x$ (or $y$ ) is interior to some leaf $W$, then $W \subseteq C_{1}, y$ sees a neighborhood of $x$ via 
$C_{1}$, and $p$ is interior to cl $S$. Since $p \in A_{1}$ and $p \notin \operatorname{bdry}(\mathrm{cl} S), p$ is in $A_{1} \cap S=B_{1}$. A similar argument holds if $x$ (or $y$ ) is interior to conv $Q$. Since neither $x$ nor $y$ is in bdry $(\mathrm{cl} S)$, the only other possibility to consider is the case in which $x, y \in \operatorname{bdry}(\operatorname{conv} Q) \sim Q \leqq \operatorname{ker}(\operatorname{cl} S)$. Then $x \in \operatorname{int}(\mathrm{cl} S), y \in \operatorname{ker}(\mathrm{cl} S), y$ sees some neighborhood of $x$ via cl $S$, and $p \in$ int (cl $S$ ). Again $p \in A_{1} \cap S=B_{1}$ and $B_{1}$ is indeed convex. Thus $S$ is the union of the convex sets $B_{i}, 1 \leqq i \leqq 4$, and the theorem is proved.

To see that the number four is best possible, let $S$ denote the set in Example 1 with its boundary deleted. Then $S$ is an open 3convex set not expressible as a union of fewer than four convex sets.

4. The general case. It remains to investigate the case for $S$ an arbitrary 3-convex subset of the plane. A decomposition of $S$ into six convex sets may be obtained from our previous results, together with Theorems 5 and 6 , which deal with the case for $(\mathrm{cl} S \sim S) \subseteq$ bdry $(\mathrm{cl} S)$.

The following result by Lawrence, Hare, and Kenelly [1, Theorem 2] will be useful:

Lawrence, Hare, Kenelly Theorem. Let $T$ be a subset of a linear space such that each finite subset $F \subseteq T$ has a $k$-partition, $\left\{F_{1}, \cdots, F_{k}\right\}$, where conv $F_{\imath} \leqq T, 1 \leqq i \leqq k$. Then $T$ is a union of $k$ convex sets.

THEOREm 5. If $\mathrm{cl} S$ is convex and $(\operatorname{cl} S \sim S) \subseteq$ bdry $(\mathrm{cl} S)$, then $S$ is a union of three or fewer convex sets. The bound of three is best possible.

Proof. Consider the collection of all intervals in bdry (cl $S)$ having endpoints in $S$ and some relatively interior point not in $S$. Each interval determines a line $L$, and by the 3-convexity of $S, L \cap S$ has exactly two components. Let $\mathscr{L}$ denote the collection of all such lines. By the Lawrence, Hare, Kenelly Theorem, without loss of generality we may assume that $\mathscr{L}$ is finite. Hence the set $\bigcup\{L \cap S: L$ in $\mathscr{L}\}$ has finitely many components, and we may order these components in a clockwise direction along bdry(cl $S)$. If $c_{i}$ denotes the $i$ th component in our ordering, let

$$
\begin{aligned}
& A^{\prime} \equiv\left\{c_{2}: i \text { odd, } i<n\right\}, \\
& B^{\prime} \equiv\left\{c_{i}: i \text { even, } i<n\right\}, \\
& C^{\prime} \equiv\left\{c_{n}\right\} .
\end{aligned}
$$

Define 


$$
\begin{aligned}
& A \equiv S \sim\left(B^{\prime} \cup C^{\prime}\right), \\
& B \equiv S \sim\left(A^{\prime} \cup C^{\prime}\right), \\
& C \equiv S \sim\left(A^{\prime} \cup B^{\prime}\right) .
\end{aligned}
$$

We assert that $A, B, C$ are convex sets whose union is $S$. The proof follows:

For $x, y$ in $A$, if $[x, y]$ contains any point of int $(\operatorname{cl} S)$, then $(x, y) \subseteq \operatorname{int}(\mathrm{cl} S) \subseteq A$, and $[x, y] \subseteq A$. Otherwise, $[x, y]$ lies in the boundary of the convex set cl $S$. If the corresponding line $L(x, y)$ is not in $\mathscr{L}$, the result is clear, so suppose $L(x, y) \in \mathscr{P}$. Then $x, y$ must lie in the same $c_{\imath}$ set for some $i$ odd, $i<n$, again giving the desired result. Hence $A$ is convex. Similarly, $B, C$ are convex. It is easy to see that $A \cup B \cup C=S$ and the proof is complete.

The surprising fact that three is best possible is illustrated by Example 2.

ExAmple 2. Let $S$ denote the set in Figure 2, where dotted lines represent segments not in $S$. Then $S$ is not expressible as a union of fewer than three convex sets.

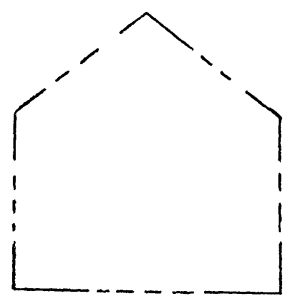

Figure 2

THEOREM 6. If (cl $S \sim S) \subseteq$ bdry (cl $S)$, then $S$ is a union of four or fewer convex sets. The number four is best possible.

Proof. We assume that $S$ is connected and $\operatorname{cl} S=\operatorname{cl}($ int $S$ ), for otherwise $S$ is a union of two convex sets. Furthermore, by the Lawrence, Hare, Kenelly Theorem, we may assume that cl $S$ has finitely many leaves, and hence card $Q=n$ is finite. Notice also that since cl $S$ is simply connnected and (cl $S \sim S) \subseteq$ bdry (cl $S), S$ is simply connected.

For the moment, suppose $3 \leqq n$. Order the points of $Q$ in a clockwise direction along bdry (conv $Q$ ), letting $W_{\imath}$ denote the leaf of cl $S$ determined by lnc points $q_{i}, q_{i+1}$ (where $n+1 \equiv 1$ ). By Valentine's results in [2], for any pair of disjoint leaves $W_{\imath}, W_{j}$ of cl $S$, the set $R \equiv \operatorname{conv} Q \cup W_{i} \cup W_{j}$ is a closed convex set. (In case there are no disjoint leaves, $n=3, W_{j}=\varnothing$, and $R \equiv \operatorname{conv} Q \cup W_{i}$ is closed and convex.) Consider the collection of intervals in bdry $R$ having end- 
points $x, y$ in $S$ and some relatively interior point $p$ not in $S$. Either such an interval is contained in one leaf, or $x \in W_{i} \cup \operatorname{conv} Q, y \in W_{j} \cup$ conv $Q$. We examine the latter case. It is clear that for an appropriate labeling, $j=i+2$, so to simplify notation, say $i=1, j=3$, and $L(x, y)$ supports $W_{2}$. Clearly not both $x, y$ can lie in conv $Q$, for then $p \in$ int $S \subseteq S$. However, we assert that either $x$ or $y$ must lie in conv $Q$ and that $W_{2} \cap S$ is convex. The proof follows:

Assume that $x$ is not an lnc point and that $x<p \leqq q_{2}<q_{3}$, where $q_{2}, q_{3}$ are the lnc points in $W_{1} \cap W_{2}, W_{2} \cap W_{3}$ respectively. Then $q_{2} \leqq y$. For $w$ in $W_{2} \cap S, w$ cannot see $x$ via $S$, so necessarily $w$ sees $y$ via $S$, by the 3-convexity of $S$. This implies that $y \leqq q_{3}$ (for otherwise $q_{3}$ could not be an lnc point for $\mathrm{cl} S$ ). Moreover, since no two points of $W_{2} \cap S$ see $x$ via $S$, the 3-convexity of $S$ together with the convexity of $W_{2}$ imply that $W_{2} \cap S$ is convex.

Here we digress briefly for future reference. The set $L(x, y) \cap S$ has two components, and by the above argument, one must lie in the interval $\left[q_{2}, q_{3}\right]$, the other in $W_{1} \sim Q$ (by our labeling). For general $W_{\imath-1}, W_{i+1}$ (disjoint if and only if $n>3$ ), we let $T_{i}$ denote the connected set of all the somewhat troublesome points $y$ in $\left[q_{i}, q_{i+1}\right] \cap S$ having the above property. That is, there exist points $x$ in exactly one of $\left(W_{i-1} \cap S\right) \sim Q,\left(W_{i+1} \cap S\right) \sim Q$ for which $[x, y] \nsubseteq S(n+1 \equiv 1)$.

Continuing the argument, delete $W_{2}$ and consider the 3-convex set $\left(S \sim W_{2}\right) \cup(S \cap L(x, y))$. Renumber the lnc points and leaves for this set so that the old $W_{1}$ and $W_{3}$ are contained in the new leaf $U_{1}$. Since we are assuming card $Q$ is finite, repeating the procedure finitely many times yields a 3-convex set $S_{0}$ having the following property: For $V_{i}, V_{j}$ disjoint leaves of $\mathrm{cl} S_{0}, x$ in $V_{i} \cap S_{0}, y$ in $V_{j} \cap S_{0}$, then $[x, y] \subseteq S_{0}$. In addition, without loss of generality we may assume that for each leaf $V_{i}$ of $\mathrm{cl} S_{0}, V_{i} \cap S_{0}$ is not convex, for otherwise, $V_{i}$ may be deleted by the above procedure.

To avoid confusion, let $Q_{0}$ denote the set of lnc points of $\mathrm{cl} S_{0}$, $Q_{0} \leqq Q$, card $Q_{0}=m \leqq n$. For $3 \leqq m$, let $V_{i}$ denote the leaf determined by lnc points $p_{\imath}, p_{i+1}$ in $Q_{0}$ (where $p_{m+1}=p_{1}$ ). For $m=2$, let $V_{1}, V_{2}$ denote the leaves of $\mathrm{cl} S_{0}$ as defined in the introduction to this paper. If $0 \leqq m \leqq 1$, let $V_{1}=V_{2}=\operatorname{cl} S_{0}$.

For each $i$, consider the collection of intervals in bdry $V_{i}$ having endpoints in $V_{i} \cap S_{0}$ and some relatively interior point not in $S_{0}$. Each interval determines a line $L$, and for $m \neq 1, L \cap V_{i} \cap S_{0}$ has exactly two components, each in bdry $V_{i}$. In case $m=1$, an obvious adjustment may be made (by deleting any ray of $L$ which contains interior points of cl $S_{0}$ ) to yield the same result. For each $i$, let $\mathscr{L}_{i}$ denote the collection of all such lines. Again using the Lawrence, Hare, Kenelly Theorem, we may assume that each $\mathscr{L}_{i}$ is finite. The set $\bigcup\left\{L \cap V_{\imath} \cap S_{0}: L\right.$ in $\left.\mathscr{L}_{i}\right\}$ has finitely many components, and we 
may order them in a clockwise direction along bdry $V_{i}$. Let $c_{i j}$ denote the $j$ th such component for $V_{i}$, and let $\mathscr{C}_{i}$ denote the collection of all the $c_{i j}$ sets corresponding to $V_{i}$. Clearly each $c_{i j}$ is either a point, an interval, or the union of two noncollinear intervals. Moreover, for $m \geqq 2$, no components for $V_{i}, V_{i+1}$ may have common points. (Such a point would necessarily be $p_{i+1}$, and if $s_{i} \in V_{i} \cap S_{0}, s_{i+1} \in V_{i+1} \cap S_{0}$ with some interior point of each of $\left[s_{i}, p_{i+1}\right],\left[p_{i+1}, s_{i+1}\right]$ not in $S_{0}$, then $s_{i}, p_{i+1}, s_{i+1}$ would be visually independent via $S_{0}$, clearly impossible.)

For each $V_{i}$, select every $c_{i 2 j}$. That is, select the members of $\mathscr{C}_{i}$ having second subscript even. No two components selected correspond to the same line, and for $m \neq 0$, we have chosen one component corresponding to each line in $\mathscr{L}_{i}$. If $m=0$, without loss of generality we may assume $\mathscr{C}_{1}$ is ordered in a clockwise direction from some point in $Q \cap \operatorname{cl} S_{0} \neq \varnothing$. In case no component has been chosen for some line $L$ in $\mathscr{L}_{1}$, then $L$ must contain points of both the first and last members of $\mathscr{C}_{1}$, and by a previous argument, one of these components must lie in $\operatorname{conv} Q$.

For $m \neq 1$, since $V_{i}$ is convex, it is easy to show that conv $\left\{c_{22 j}: 1 \leqq j\right\}$ is a subset of $S_{0}$ (and this is certainly true even if cl $S_{0}$ is convex). We will prove that $B_{0} \equiv \operatorname{conv}\left\{c_{i 2 j}: 1 \leqq i \leqq m, 1 \leqq j\right\}$ is in $S_{0}$ and hence in $S$. If $\mathrm{cl} S_{0}$ is convex (or empty) the result is immediate, so assume cl $S_{0}$ has at least one lnc point. For convenience, in case $\mathrm{cl} S_{0}$ has only one lnc point, call it $p_{2}$, and let $V_{1}=V_{2}$ follow $p_{2}$ in our clockwise ordering.

Recall that $V_{i} \cap S_{0}$ is not convex for any $i$, so no $\mathscr{C}_{i}$ is empty. Let $c_{0}$ denote the last member of $\mathscr{C}_{1}$ selected, $x$ the last point of cl $c_{0}$ (relative to our ordering). If $x \neq p_{2}$, let $L=L\left(x, p_{2}\right)$. Otherwise, by the 3-convexity of $S_{0}, c_{0}=\left\{p_{2}\right\}$, and in this case let $L$ denote the corresponding member of $\mathscr{L}_{1}$. Let $L_{1}, L_{2}$ be the open halfspaces determined by $L$, with $Q_{0} \leqq \operatorname{cl~} L_{1}$. Since $p_{2}$ is an lnc point of $S_{0}$ and $S_{0}$ is 3-convex, it is clear that at most one member of $\mathscr{C}_{2}$, namely $c_{21}$, may contain points in $L_{2}$. We assert that $c_{0}$ sees $c_{22}$ via $S_{0}$. The proof follows:

In case $L \in \mathscr{L}_{1}, L \cap V_{1} \cap S_{0}$ has two components, each in bdry $V_{1}$, and one of these must be $\left\{p_{2}\right\}$. Then by the 3-convexity of $S_{0}, c_{22} \subseteq L_{1}$ and $c_{0}$ sees $c_{22}$ via $S_{0}$. Otherwise, $c_{0} \sim\{x\} \subseteq L_{1}$. If $x \notin S_{0}$, then since $c_{22} \subseteq \mathrm{cl} L_{1}$, it is clear that $c_{0}$ sees $c_{22}$ via $S_{0}$. If $x \in S_{0}$ and $p_{2} \in S_{0}$, then again the result is clear. If $x \in S_{0}$ and $p_{2} \notin S_{0}$, then $c_{22} \leqq L_{1}$ and $c_{0}$ sees $c_{22}$ via $S_{0}$, finishing the argument.

In case $V_{1}, V_{2}$ are the only leaves for $\mathrm{cl} S_{0}, V_{1} \neq V_{2}$, then repeating the argument for the last member of $\mathscr{C}_{2}$ and $c_{12}$ and using the fact that $S_{0}$ is simply connected, we have $B_{0} \subseteq S_{0} \subseteq S$. (If $V_{1}=V_{2}$, the result is immediate.) Otherwise, $3 \leqq m$ and an inductive argument may be used to show that $B_{0}$ is in $S$. 
Using Valentine's results, write cl $S$ as a union of three or fewer convex sets $A_{j}, j=1,2,3$, where for $n$ odd

$$
\begin{aligned}
& A_{1} \equiv \bigcup\left\{W_{i}: i \text { odd, } i<n\right\} \cup \operatorname{conv} Q, \\
& A_{2} \equiv \bigcup\left\{W_{i}: i \text { even, } i<n\right\} \cup \operatorname{conv} Q, \\
& A_{3} \equiv W_{n} \cup \operatorname{conv} Q,
\end{aligned}
$$

and for $n$ even

$$
\begin{aligned}
& A_{1} \equiv \bigcup\left\{W_{i}: i \text { odd, } i \leqq n\right\} \cup \operatorname{conv} Q, \\
& A_{2}=\bigcup\left\{W_{i}: i \text { even, } i \leqq n\right\} \cup \operatorname{conv} Q, \\
& A_{3}=\varnothing .
\end{aligned}
$$

Define $B_{j} \equiv S \cap\left[A_{j} \sim\left((\right.\right.$ bdry $\left.\left.S) \cap B_{0}\right)\right], j=1,2,3$.

Recall the $T_{i}$ sets defined previously, $T_{i} \leqq\left[q_{i}, q_{i+1}\right] \leqq W_{\imath}, 1 \leqq i \leqq n$. To simplify notation, let $L_{i}=L\left(q_{i}, q_{i+1}\right)$, and define sets $F_{i}, G_{i}$ in the following manner: For $i$ even, let $F_{i}=T_{i}$ if points from both components of $L_{\imath} \cap S$ are in $B_{1}, F_{i}=\varnothing$ otherwise. Similarly for $i$ odd, let $F_{i}=T_{i}$ if points from both components of $L_{i} \cap S$ are in $B_{2}, F_{i}=\varnothing$ otherwise. For $i=1, i=n-1$, let $G_{i}=T_{i}$ if points from both components of $L_{i} \cap S$ are in $B_{3}, G_{i}=\varnothing$ otherwise. By previous remarks, at least one of $G_{1}, F_{1}$ is empty, and at least one of $G_{n-1}, F_{n-1}$ is empty.

Define

$$
\begin{aligned}
& D_{1} \equiv B_{1} \sim \bigcup\left\{F_{\imath}: i \text { even }\right\} \\
& D_{2} \equiv B_{2} \sim \bigcup\left\{F_{i}: i \text { odd }\right\}, \\
& D_{3} \equiv B_{3} \sim \bigcup\left\{G_{i}, G_{n-1}\right\}
\end{aligned}
$$

Finally, letting $P=\left\{F_{i} \cap F_{j}: 1 \leqq i<j \leqq n\right\} \cup\left\{G_{i} \cap F_{j}: i=1, n-1\right.$, $1 \leqq j \leqq n\}$, define $D_{0} \equiv \operatorname{conv}\left(B_{0} \cup P\right)$. We assert that the sets $D_{j}$, $0 \leqq j \leqq 3$, are convex sets whose union is $S$. The proof follows:

Suppose that one of the sets $D_{1}, D_{2}, D_{3}$, say $D_{1}$, is not convex to obtain a contradiction. Choose $x, y$ in $D_{1}$ for which $[x, y] \nsubseteq D_{1}$. It is clear that $[x, y] \subseteq$ bdry $\left(\operatorname{cl} D_{1}\right)=$ bdry $A_{1}$. Furthermore, $x, y$ cannot both belong to $W \sim Q$ for any leaf $W$ of $\mathrm{cl} S$, for otherwise they would belong to the same leaf of $\mathrm{cl} S_{0}$, and one of $x, y$ would lie in (bdry $S) \cap B_{0}$ and hence not in $D_{1}$, a contradiction. Employing a previous argument, the set $L(x, y) \cap S$ has two components, each having points in $B_{1}$, and one of these components is the set $\left[q_{i}, q_{\imath+1}\right] \cap$ $S=T_{2}$ for some $i$ even $(n+1 \equiv 1)$. Let $R_{i}$ denote the other component of $L(x, y) \cap S$. If $R_{i} \cap B_{0} \neq \varnothing$, then $R_{i}, T_{i}$ would lie on the boundary of a leaf of $\mathrm{cl} S_{0}, R_{i} \subseteq B_{0}, T_{i} \subseteq B_{1}$, and $[x, y] \leqq T_{i} \leqq D_{1}$, a contradiction. Thus $R_{i} \cap B_{0}=\varnothing$ and $R_{i} \subseteq D_{1}$. However, this implies that one of $x, y$ must lie in $F_{i}$ and not in $D_{1}$, again a contradiction. Our assumption is false and $D_{1}$ is convex. Similarly $D_{2}, D_{3}$ are convex, 
and clearly each is a subset of $S$.

It remains to show that the convex set $D_{0}$ lies in $S$. Examining the set $P$, if $F_{i} \cap F_{j} \neq \varnothing$ for some $i \neq j$ (or if $G_{i} \cap F_{j} \neq \varnothing$ ), then $F_{i}=T_{i}, F_{j}=T_{j}$, for an appropriate labeling $j=i+1$, and $F_{i} \cap F_{i+1}=$ $\left\{q_{i+1}\right\} \subseteq S$. We will show that for each $z$ in $B_{0},\left[q_{i+1}, z\right] \subseteq S$. The proof follows:

We have seen that $W_{\imath} \cap S, W_{i+1} \cap S$ are both convex, so for every $z$ in one of these sets, $\left[q_{i+1}, z\right] \subseteq S$. Moreover, we assert that the components of $L\left(q_{i}, q_{i+1}\right) \cap S, L\left(q_{i+1}, q_{i+2}\right) \cap S$ not in conv $Q$, call them $R_{i}, R_{i+1}$, are disjoint from $B_{0}$ : If $R_{i} \cap B_{0} \neq \varnothing$, then by an earlier argument, $R_{i} \subseteq B_{0}, \quad T_{i} \cap B_{0}=\varnothing, \quad T_{i} \subseteq D_{1} \cap D_{2} \cap D_{3}$, and $F_{i}=\varnothing$, a contradiction. Hence for $z$ in $B_{0} \sim\left(W_{i} \cup W_{i+1}\right),\left(q_{i+1}, z\right) \subseteq$ int $S$, and $\left[q_{i+1}, z\right] \subseteq S$ whenever $z \in B_{0}$, the desired result.

Certainly for $q_{i}, q_{j}, q_{k}$ in $P \subseteq S$, conv $\left\{q_{i}, q_{j}, q_{k}\right\} \subseteq S$.

By Carathéodory's theorem in the plane, to prove that $D_{0} \equiv$ conv $\left(B_{0} \cup P\right)$ is in $S$, it is sufficient to show that the convex hull of any three points of $B_{0} \cup P$ is in $S$, and from the remarks above, clearly we need only show $\operatorname{conv}\left\{q_{i}, q_{j}, z\right\} \subseteq S$ for $q_{i}, q_{j}$ in $P, z$ in $B_{0}$. However, since $S$ is simply connected and bdry $\left(\operatorname{conv}\left\{q_{i}, q_{j}, z\right\}\right) \subseteq S$, conv $\left\{q_{i}, q_{j}, z\right\} \subseteq S$ and $D_{0} \subseteq S$, the desired result.

Finally, by inspection, each $F_{i} \neq \varnothing$ fails to belong to at most one of the sets $D_{1}, D_{2}, D_{3}$. Points in intersecting $F_{i}$ sets are in $D_{0}$, so $\bigcup\left\{D_{j}: 0 \leqq j \leqq 3\right\}=S$ and the argument for $3 \leqq \operatorname{card} Q$ is complete.

To finish the proof, we must examine the cases for $0 \leqq$ card $Q \leqq 2$. If $\operatorname{card} Q=2$ or if card $Q=1$ and $S \sim Q$ is connected, then let $W_{1}, W_{2}$ denote the corresponding leaves of $\mathrm{cl} S$, and use a simplified version of the previous proof to define $B_{0}, B_{1}, B_{2}$. If one of $B_{1}, B_{2}$, say $B_{1}$, is not convex, then letting $T=W_{1} \cap W_{2} \cap S, W_{2} \cap S=B_{2}$ is convex, $T \subseteq B_{2}$, and $B_{0}, B_{1} \sim T, B_{2}$ are the desired convex sets.

In case card $Q=1$ and $S \sim Q$ is not connected, then for $W_{1}, W_{2}$ the corresponding leaves of $\mathrm{cl} S$, each of $W_{1} \cap S, W_{2} \cap S$ is convex. For card $Q=0$, the result follows from Theorem 5 , and the proof of Theorem 6 is complete.

The number four in Theorem 6 is best possible, as the following example illustrates.

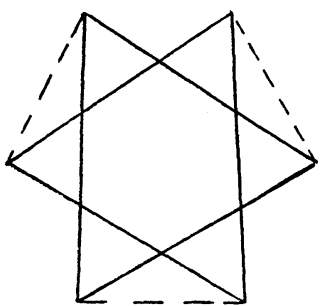

Figure 3 
ExAmple 3. Let $S$ denote the set in Figure 3, where dotted segments are in bdry (cl $S) \sim S$. Then $S$ is a union of no fewer than four convex sets.

At last, using Theorem 6, we have a decomposition theorem for $S$ an arbitrary 3 -convex subset of the plane.

THEOREM 7. The set $S$ is a union of six or fewer convex sets. The result is best possible.

Proof. By earlier comments, we may assume that $S$ is connected, $\operatorname{cl} S=\operatorname{cl}($ int $S$ ), and $Q$ is finite. Furthermore, we assume int $(\operatorname{cl} S) \sim$ $S \neq \varnothing$, for otherwise the result is an immediate consequence of Theorem 6. Let $T \equiv S \cup$ bdry (cl $S)$, and let $L$ be the line containing cl $T \sim T$ described in Theorem 2 or Theorem 3 (whichever is appropriate). Clearly $L$ may be chosen to contain an lnc point $q$ of cl $S$. If $L_{1}, L_{2}$ are the corresponding open halfspaces, then each of $T_{1} \equiv$ $\operatorname{cl}\left(T \cap L_{1}\right)=\operatorname{cl}\left(S \cap L_{1}\right), T_{2} \equiv \operatorname{cl}\left(T \cap L_{2}\right)=\operatorname{cl}\left(S \cap L_{2}\right)$ is 3-convex.

Define $S_{i} \equiv T_{i} \cap S, i=1,2$, . We assert that each $S_{i}$ is 3-convex: For $x, y, z$ in $S_{1}=T_{1} \cap S$, assume $[x, y]$ lies in the 3-convex set $S$ to show $[x, y] \subseteq S_{1}$. If $x$ or $y$ is in $L_{1}$, then certainly $(x, y) \subseteq L_{1} \cap S \subseteq T_{1}$, and $[x, y] \subseteq S_{1}$. If $x, y$ are on $L$, then since no lnc points of the closed set $T_{1}$ are on $L, x, y$ lie in the same leaf of $T_{1}$, and $[x, y] \subseteq T_{1} \cap S=S_{1}$. Thus $S_{1}$ is 3-convex. Similarly $S_{2}$ is 3-convex. Moreover, $\left(\operatorname{cl} S_{i} \sim S_{i}\right) \subseteq$ bdry $\left(\mathrm{cl} S_{i}\right), i=1,2$.

Using Theorem 6, we will show that each $S_{i}$ is a union of three convex sets: By the proofs of Theorems 2 and $3, \mathrm{cl} S_{1}=T_{1}$ is a union of two convex sets $A_{1}, A_{2}$, and each $A_{i}$ may be considered a subset of an appropriate $C_{j}$ set, $1 \leqq j \leqq 3$, where the $C_{j}$ sets are those described in Valentine's paper with $\mathrm{cl} S=C_{1} \cup C_{2} \cup C_{3}$. In case $T_{1}$ has one leaf or an even number of leaves, then clearly the proof of Theorem 6 may be used to write $S_{1}$ as a union of three convex sets. If $T_{1}$ has $n$ leaves for $n$ odd, $n>1$, let $V$ be the leaf of $T_{1}$ bounded by $L$, $q \in Q \cap L \subseteq A_{1} \cap A_{2}$. Order the lnc points of $T_{1}$ in a clockwise direction so that $V$ is determined by $q_{n}, q_{1}$, and let $U_{n}, U_{n+1}$ denote the closed subsets of $V$ bounded by $L\left(q_{n}, q\right), L\left(q, q_{1}\right)$ respectively. Treating $U_{1}, \cdots, U_{n}, U_{n+1}$ as leaves of $T_{1}, U_{i}$ determined by $\operatorname{lnc}$ points $q_{i}, q_{i+1}$, $1 \leqq i<n$, the proof of Theorem 6 may be applied to write $S_{1}$ as a union of three convex sets. (Of course, in defining $B_{0}$, points of $V$ in $S_{0}$ belong to the same leaf of $S_{0}$.)

By a parallel argument $S_{2}$ is a union of three convex sets, and $S=S_{1} \cup S_{2}$ is a union of six or fewer convex sets, finishing the proof of the theorem.

Our final example shows that the bound of six in Theorem 7 is 
best possible.

EXAMPLE 4. Let $S$ be the set in Figure 4, with dotted segments in bdry $(\operatorname{cl} S) \sim S$ and $p \in \operatorname{int}(\operatorname{cl} S) \sim S$. Then $S$ cannot be expressed as a union of fewer than six convex sets.

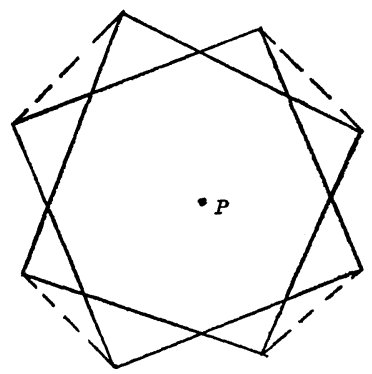

Figure 4

\section{REFERENCES}

1. J. F. Lawrence, W. R. Hare, and John W. Kenelly, Finite unions of convex sets, Proc. Amer. Math. Soc., 34 (1972), 225-228.

2. F. A. Valentine, A three point convexity property, Pacific J. Math., 7 (1957), 12271235 .

Received May 15, 1973.

The UNIVERSity OF OKLAHOMA 



\section{PACIFIC JOURNAL OF MATHEMATICS}

\section{EDITORS}

RICHARD ARENS (Managing Editor)

University of California

Los Angeles, California 90024

R. A. Beaumont

University of Washington

Seattle, Washington 98105
J. DugundJI

Department of Mathematics

University of Southern California

Los Angeles, California 90007

D. Gilbarg and J. Milgram

Stanford University

Stanford, California 94305

\section{ASSOCIATE EDITORS}

E. F. BECKENBACH

B. H. NeUMANN

F. WOLF

K. YosHIDA

\section{SUPPORTING INSTITUTIONS}

UNIVERSITY OF BRITISH COLUMBIA

CALIFORNIA INSTITUTE OF TECHNOLOGY

UNIVERSITY OF CALIFORNIA

MONTANA STATE UNIVERSITY

UNIVERSITY OF NEVADA

NEW MEXICO STATE UNIVERSITY

OREGON STATE UNIVERSITY

UNIVERSITY OF OREGON

OSAKA UNIVERSITY
UNIVERSITY OF SOUTHERN CALIFORNIA

STANFORD UNIVERSITY

UNIVERSITY OF TOKYO

UNIVERSITY OF UTAH

WASHINGTON STATE UNIVERSITY

UNIVERSITY OF WASHINGTON

$\stackrel{*}{*} \stackrel{*}{*} \stackrel{*}{*}$ AMERICAN MATHEMATICAL SOCIETY




\section{Pacific Journal of Mathematics}

\section{Vol. 53, No. $1 \quad$ March, 1974}

Martin Bartelt, Strongly unique best approximates to a function on a set, and a finite

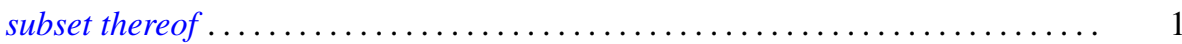

S. J. Bernau, Theorems of Korovkin type for $L_{p}$-spaces $\ldots \ldots \ldots \ldots \ldots \ldots \ldots \ldots \ldots$

S. J. Bernau and Howard E. Lacey, The range of a contractive projection on an

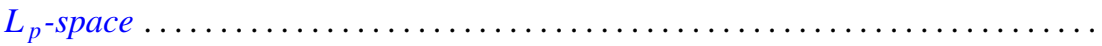

Marilyn Breen, Decomposition theorems for 3-convex subsets of the plane ......... Ronald Elroy Bruck, Jr., A common fixed point theorem for a commuting family of

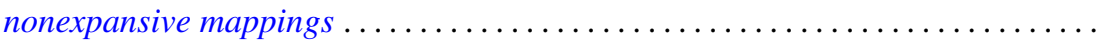

Aiden A. Bruen and J. C. Fisher, Blocking sets and complete $k$-arcs . . . . . . . 73

R. Creighton Buck, Approximation properties of vector valued functions . ......... 85

Mary Rodriguez Embry and Marvin Rosenblum, Spectra, tensor products, and

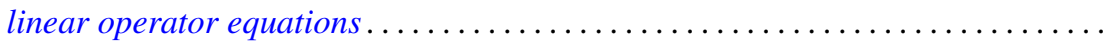

Edward William Formanek, Maximal quotient rings of group rings . . . . . . . . . 109

Barry J. Gardner, Some aspects of T-nilpotence . . . . . . . . . . . . . . . 117

Juan A. Gatica and William A. Kirk, A fixed point theorem for $k$-set-contractions

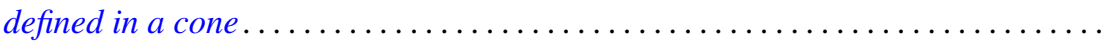

Kenneth R. Goodearl, Localization and splitting in hereditary noetherian prime

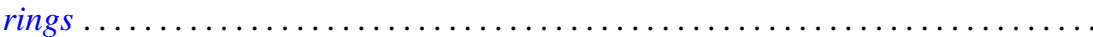

James Victor Herod, Generators for evolution systems with quasi continuous

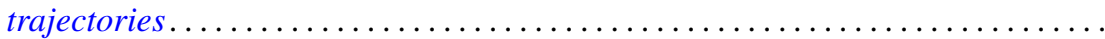

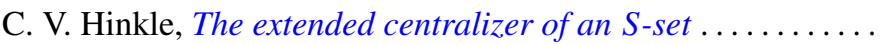

I. Martin (Irving) Isaacs, Lifting Brauer characters of p-solvable groups . . .

Bruce R. Johnson, Generalized Lerch zeta function ...........

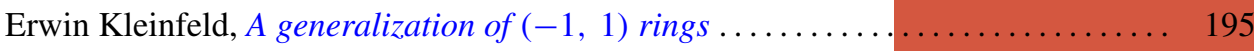

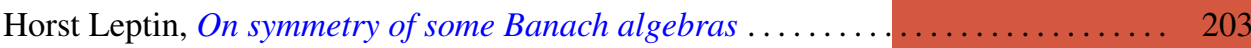

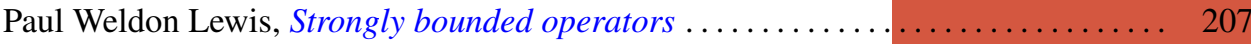

Arthur Larry Lieberman, Spectral distribution of the sum of self-adjoint

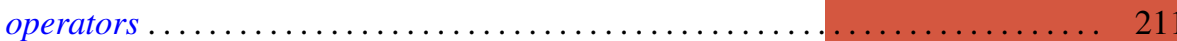

I. J. Maddox and Michael A. L. Willey, Continuous operators on paranormed spaces and matrix transformations

James Dolan Reid, On rings on groups ........................... 229

Richard Miles Schori and James Edward West, Hyperspaces of graphs are Hilbert cubes.

William H. Specht, A factorization theorem for p-constrained groups ...

Robert L Thele, Iterative techniques for approximation of fixed points of certain nonlinear mappings in Banach spaces ...............

Tim Eden Traynor, An elementary proof of the lifting theorem

Charles Irvin Vinsonhaler and William Jennings Wickless, Completely decomposable groups which admit only nilpotent multiplications .

Raymond O’Neil Wells, Jr, Comparison of de Rham and Dolbeault cohomology for

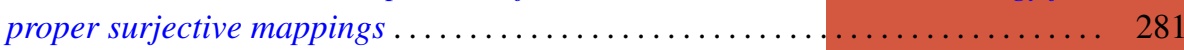

David Lee Wright, The non-minimality of induced central representations . . . . . 301 